\title{
Pelvic Inflammatory Disease: A review of presentations to our service
}

\section{Dr Kirsten Michie \& Dr Frances Hunt, Royal Liverpool Hospital}

\section{Introduction}

- Pelvic inflammatory disease (PID) is an important presentation to sexual health services, and can be a complication of the sexually transmitted infections Chlamydia trachomatis and Neisseria gonorrhoea.

- The diagnosis should be considered in any young woman presenting with recent onset bilateral lower abdominal pain, with tenderness on bimanual examination, in whom pregnancy has been excluded.

- Complications of PID include tubal infertility.

\section{Aims}

- We sought to review the presentation and management of PID in women attending our service.

\section{Methods}

- We used the SHHAPT code C5A to identify women diagnosed with PID between 01/06/15 - 30/11/15.

- A retrospective case note review was performed; collecting demographic data and details regarding presentation and management.

\section{Results}

- 50 cases were identified.

- Age range: 17 - 40 years (median 23.5 years).

\section{Presenting Symptoms}

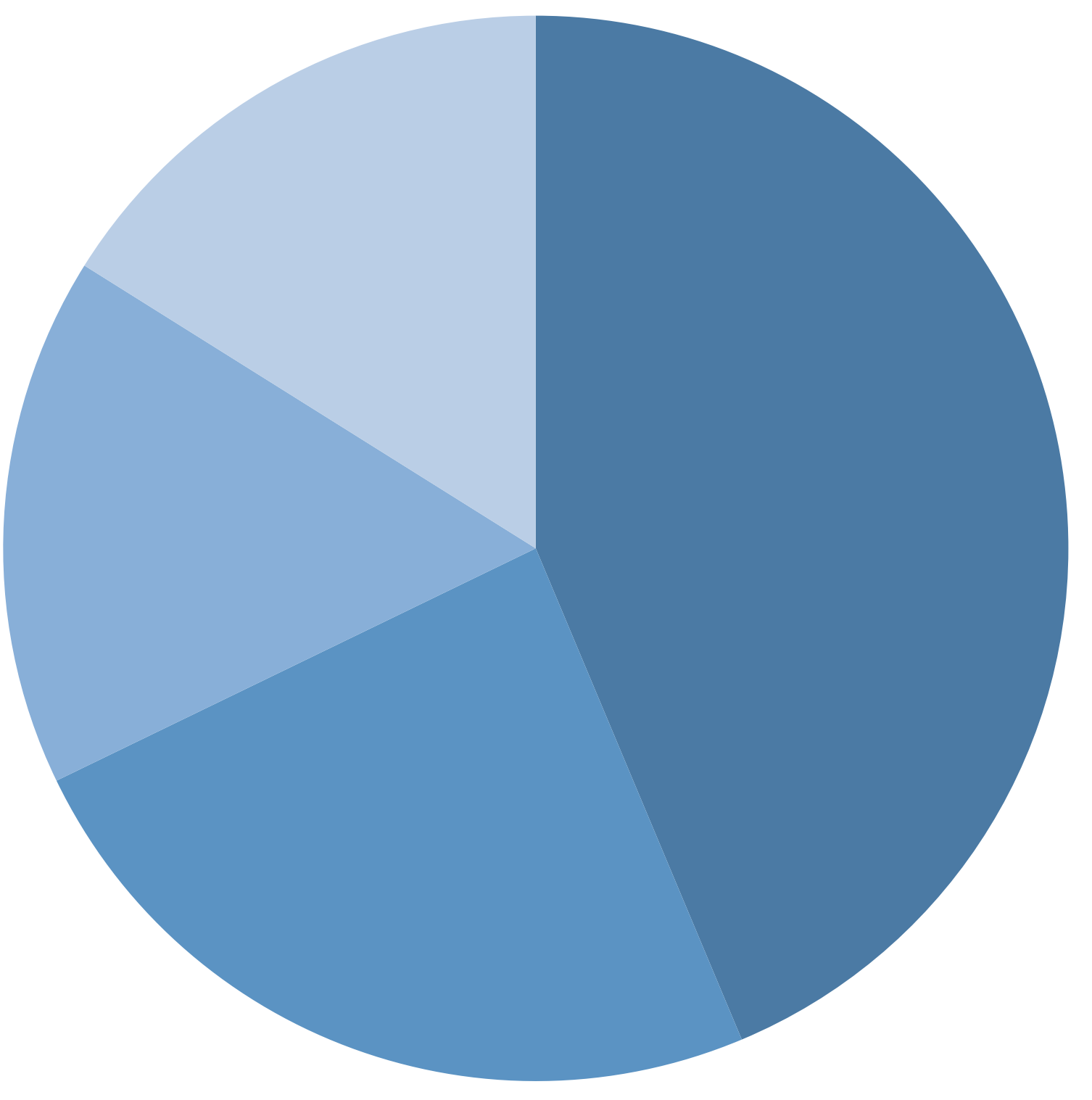

\section{Sexual History}

- 40/50 women reported having a regular male partner.

- The majority of women (42/50) had had one partner only in the preceding 3 months.

\section{STI Screening}

- Dyspareunia $(14 / 50)$

Bleeding irregularities $(14 / 50)$
- $4 / 50$ women tested positive for chlamydia, all of whom were aged less than 25.

- No other sexually transmitted infections were identified.

- The rate of chlamydia amongst women aged less than 25 treated for PID was $4 / 31$ (12.9\%).

\section{Follow up}

- Less than half of women (19/50) attended for follow up.

- There was documentation of the regular male partner attending for treatment in only $14 / 40$ cases.

\section{Conclusions}

- Our project reflects chlamydia as an important cause of PID in younger women.

- This supports the latest BASHH guidance to offer repeat chlamydia screening to under 25's to help identify reinfections and prevent complications such as PID. 